\title{
Using corticomuscular and intermuscular coherence to assess cortical contribution to ankle plantar flexor activity during gait
}

Jensen, Peter; Frisk, Rasmus; Spedden, Meaghan Elizabeth; Geertsen, Svend Sparre;

Bouyer, Laurent J; Halliday, David M; Nielsen, Jens Bo

Published in:

Journal of Motor Behavior

DOI:

10.1080/00222895.2018.1563762

Publication date:

2019

Document version

Peer reviewed version

Citation for published version (APA):

Jensen, P., Frisk, R., Spedden, M. E., Geertsen, S. S., Bouyer, L. J., Halliday, D. M., \& Nielsen, J. B. (2019).

Using corticomuscular and intermuscular coherence to assess cortical contribution to ankle plantar flexor activity during gait. Journal of Motor Behavior, 51(6), 668-680. https://doi.org/10.1080/00222895.2018.1563762 
Using corticomuscular and intermuscular coherence to assess cortical contribution to ankle plantar flexor activity during the forward propulsion of gait

Peter Jensen ${ }^{1}$, Rasmus Frisk ${ }^{1,3}$, Meaghan Elizabeth Spedden ${ }^{1}$, Svend Sparre Geertsen ${ }^{1,2}$, Laurent J. Bouyer ${ }^{4}$, David M. Halliday ${ }^{5}$ and Jens Bo Nielsen ${ }^{1,3}$

${ }^{1}$ Department of Nutrition, Exercise and Sports, University of Copenhagen, Copenhagen, Denmark

${ }^{2}$ Department of Neuroscience, University of Copenhagen, Copenhagen, Denmark ${ }^{3}$ Elsass Institute, Charlottenlund, Denmark ${ }^{4}$ CIRRIS-Department of Rehabilitation, Universite Laval, Quebec City, Canada ${ }^{5}$ Department of Electronic Engineering, University of York, UK

Running title: Corticomuscular and EMG-EMG coherence during gait Keywords: Coherence; gait, EMG, Cortex, spinal motor neurons, directionality;

\section{Proof and correspondence to:}

Peter Jensen

Department of Nutrition, Exercise and Sports

University of Copenhagen,

The Panum Institute 33.3

Blegdamsvej 3, 2200 Copenhagen N, Denmark.

Phone: +45 272037 33, e-mail:ptramp@gmail.com 


\begin{abstract}
Propulsion during human bipedal walking is produced to a large extent by ankle plantar flexor muscle activity during push-off at the end of the stance phase. The contribution from the motor cortex to this phase of locomotion is not well understood. The present study used coherence and directionality analyses to explore whether the motor cortex contributes to this activity. Eleven healthy adult subjects (age 24.9 \pm 2.8 ) walked on a treadmill at $3.6 \mathrm{~km} / \mathrm{h}$, while EEG was recorded over the leg motor cortex area and EMG was recorded from the medial gastrocnemius and soleus muscles. Corticomuscular and intermuscular coherence were calculated from pair-wise recordings. Significant EEG-EMG and EMG-EMG coherence in the beta and gamma frequency bands was found throughout the stance phase in the population average from all subjects. Analysis of directionality revealed that EEG activity preceded EMG activity throughout the stance phase until the time of push-off.

Quantification of EEG-EMG coherence showed that the largest coherence was found towards the end of stance just prior to push-off.

We interpret these findings to suggest that the motor cortex contributes to ankle plantar flexor muscle activity and forward propulsion during gait.
\end{abstract}




\section{Introduction}

The evolutionary success of human bipedal gait is evident from its daily practice by 7 billion people all over the world, regardless of whether it ensures a more economical way of walking and running Bramble and Lieberman 2004, Steudel-Numbers 2001, frees the hands for

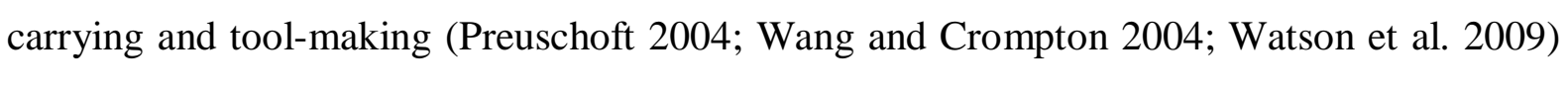
or some other advantage. A hallmark of human bipedal gait is that forward propulsion is generated by moving the center of gravity slightly in front of the supporting leg with the help of relatively little activity in plantar flexor muscles during the stance phase and at push-off

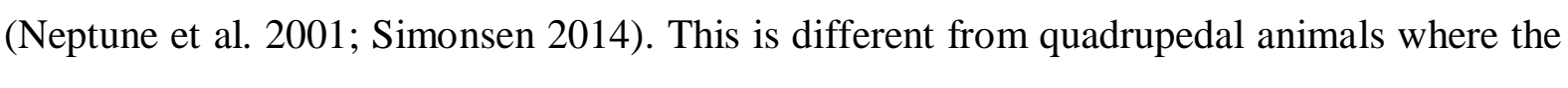
center of gravity is placed well in front of the hind limbs throughout the gait cycle, which allows that activity in hip extensors may produce significant forward propulsion during most of the stance phase Shapiro and Jungers 1994, Trank et al. 1996. This difference in the kinematics of human gait must have required significant re-organization of the central networks involved in the generation of locomotion when human gait first evolved Cappellini et al. 2010, Nielsen 2003.

The basic rhythmicity of quadrupedal locomotion is generated by spinal networks, which may function relatively independently of supraspinal control Kiehn 2016. In primates and especially humans, spinal networks appear to depend more on supraspinal input and lesion of the leg area of the motor cortex leaves the individual with severe paresis of leg muscles and strongly reduced capacity for locomotion \begin{tabular}{|l|l|l|}
\hline Dietz 2010, Eidelberg et al. 1981, & Fedirchuk et al. \\
\hline
\end{tabular} 1998. Electrophysiological and imaging studies have also provided evidence that the motor cortex contributes to the activation of leg muscles during gait with most interest devoted to the control of the ankle dorsiflexor muscles in the swing phase Capaday et al. 1999; \begin{tabular}{|l|l|l|}
\hline Hamacher et al. 2015; & la Fougere et al. 2010; Petersen et al. 1998: Petersen et al. 2001, \\
\hline
\end{tabular}

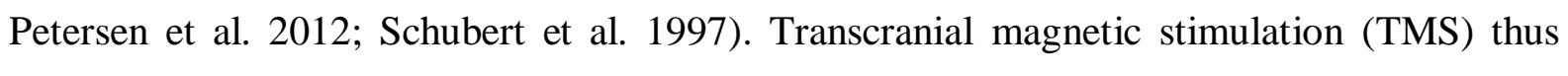
produces significant activation of the dorsiflexor muscles in the swing phase, partly through the monosynaptic corticomotoneuronal pathway Petersen et al. 1998. Brief disruption of activity in the motor cortex produces a drop in the ankle dorsiflexor EMG activity, which likely reflects a reduction in the corticospinal drive to the muscle Petersen et al. 2001. As direct evidence of this drive, corticomuscular coherence has been demonstrated between the leg area of the motor cortex and EMG activity in ankle dorsiflexor muscles in a large part of the swing phase during gait Petersen et al. 2012. 
One reason for the dominant interest in the ankle dorsiflexor muscles is that paresis of these muscles and the resulting foot drop during gait are among the most common symptoms following stroke and other lesions of the corticospinal pathways Barthelemy et al. 2013, Everaert et al. 2010. Ankle plantar flexor muscles on the other hand depend significantly on sensory feedback mechanisms in the maintenance of their activity when the foot is on the ground in the stance phase of gait Christensen et al. 2000 Nielsen and Sinkjaer 2002 Zehr and Stein 1999]. It has also been argued from observations of motor evoked potentials elicited by TMS that the motor cortex shows little interest in ankle plantar flexors during gait Capaday et al. 1999.

However, Petersen et al. (1998) demonstrated that the monosynaptic corticomotoneuronal excitation of H-reflexes in ankle plantar flexor muscles is modulated during the stance phase of gait with large excitation appearing prior to push-off. This may suggest that corticospinal drive contributes to the ankle plantar flexor activity during stance and may play a role in forward propulsion. If so, this may explain impaired push-off and forward propulsion in people with stroke Brouwer et al. 2009, Knarr et al. 2013, spinal cord injury Pepin et al. 2003a: Pepin et al. 2003b) and cerebral palsy (Geertsen et al. 2015, Hegarty et al. 2016). It was consequently the purpose of the present study to explore whether motor cortex and corticospinal tract contribute to plantar flexor muscle activity in the stance phase of walking by calculating corticomuscular coherence between EEG from the leg area of the motor cortex and EMG from ankle plantar flexors during treadmill gait. We used coherence analysis to quantify the relationship between EEG and EMG signals, and a non-parametric directionality analysis Halliday 2015 to quantify the contribution of descending and ascending activity to this coherence.

\section{Methods}

Subjects and ethics approval

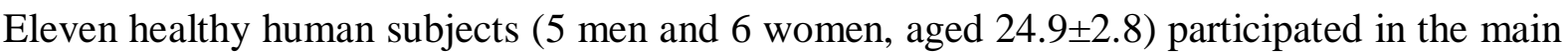
study and ten healthy human subjects ( 4 men and 6 women, aged $26.3 \pm 4.5$ ) participated in a control experiment designed to determine the cortical location of optimal corticomuscular coherence. Written consent was obtained from all subjects before the start of the study. The study was approved by the local ethics committee for the greater Copenhagen area (ref. 26397), and was reported to the Danish Data Protection Agency. The study was in agreement with the consolidated act nr. 69 of January $8^{\text {th }} 1999$ and was performed in accordance with the Helsinki II declaration. 


\section{Experimental procedure}

Subjects walked on a motorized treadmill (Technogym D140, Gambettola (FO), Italy) at 3.6 $\mathrm{km} / \mathrm{h}$ (Fig. 1). An electrogoniometer Noel et al. 2008 was attached on the right ankle of the subject and a pressure sensitive sensor was placed underneath the heel. All recordings were obtained during 5 minutes of regular walking. In the control experiment, subjects walked with an average speed of $2.2 \pm 0.1 \mathrm{~km} / \mathrm{h}$.

Data collection

All ankle angles were collected by an electrogoniometer. The timing of the heel contact during walking was indicated by a pressure sensitive sensor underneath the right foot and all data were synchronized with heel contact.

For the main study, surface EMG from medial gastrocnemius (MG) and soleus (SOL) muscles was collected using standard silver/silver chloride EMG electrodes (Ambu Blue Sensor NF-00-S/12, Ambu, Ballerup, Denmark; recording area $0.5 \mathrm{~cm}^{2}$, inter electrode distance $2 \mathrm{~cm}$ ). After careful palpation of the muscles, the skin was shaved and cleaned and the electrodes were placed on the middle part of the muscle belly.

Surface EEG was collected over the leg area of the motor cortex $(\mathrm{Cz})$ using a standard cup electrode. $\mathrm{Cz}$ was determined as the intersection of a line connecting the external occipital protuberance and glabella and a line connecting the two earlobes. The reference electrode was put $5 \mathrm{~cm}$ in front of the electrode at $\mathrm{Cz}$. The ground electrode consisted of a lead plate with a wet cloth on the elbow. To minimize artifacts during walking the subjects were instructed to relax their face and to minimize eye blinks and swallowing during the experiment. Likewise, the arms were resting on a handrail in front of the subject. Artifacts related to heel strike were avoided in the analysis by using periods starting $200 \mathrm{~ms}$ after heel strike and there was no heel strike in the periods being analyzed in order to minimize an influence from mechanical artifacts related to heel contact Petersen et al. 2012. Also, it was checked that no coherence was present between Cz-TA in this time-period.

In the control experiment surface EEG from 64 channels was collected (ActiveTwo, BioSemi, Amsterdam, The Netherlands) using acquisition software ActiView (version 6.05). Active EEG electrodes were mounted in a 64 channel headcap (Headcap BioSemi, the Netherlands). Surface EMG from MG and SOL was collected using two pairs of bipolar active EMG electrodes (interelectrode distance, $1.5 \mathrm{~cm}$ ). As per BioSemi's design, the ground electrode during acquisition was formed by the Common Mode Sense active electrode and 
the Driven Right Leg passive electrode. Data were filtered (high pass filter of $1 \mathrm{~Hz}$; low pass filter of $1000 \mathrm{~Hz}$ ), sampled at $2048 \mathrm{~Hz}$ and stored on a computer for later offline analysis. The setup in the control experiment did not allow simultaneous collection of goniometer data and hence the data obtained in the control experiment were only used to confirm the electrode placement in the main study.

\section{Data processing}

All analog signals (from the main study) were digitized on-line and sampled at $1000 \mathrm{~Hz}$. Before being digitized the EEG and EMG signal were high pass filtered at $5 \mathrm{~Hz}$ and low pass filtered at $1000 \mathrm{~Hz}$. We adopted a unified framework for analysis of EEG and EMG signals. Time domain and frequency domain estimates of correlation between the signals were obtained through spectral estimates constructed by averaging over short segments of data. Visual inspection of the EMG signal and the goniometer determined the segments used. The timing of these sections was specified using an offset with respect to the time of heel contact, obtained from the footswitch Thus, the analysis was based on averaging over short segments that had the same relative timing with respect to heel contact in each gait cycle. Prior to spectral analysis EMG signals were full wave rectified to suppress information relating to action potential waveform shape Halliday and Farmer 2010. All rectified EMG signals were normalized to have unit variance within each segment (Halliday and Rosenberg 2000, the consequences of this step are that spectral estimates reflect changes in relative power rather than absolute power. In addition, cumulant density, or cross covariance, time domain estimates of correlation are easier to compare across different parts of the step cycle where the EMG has different amplitudes. Coherence estimates are unaffected by this normalization process. Normalized EEG and rectified and normalized EMG signals are assumed to be realizations of stationary zero mean time series denoted by $\mathrm{x}$ and $\mathrm{y}$. Discrete Fourier transform was applied to the signals to estimate the average autospectra $f_{\mathrm{xx}}(\lambda)$ and $f_{\mathrm{yy}}(\lambda)$ and cross-spectra $f_{\mathrm{xy}}(\lambda)$. Frequency domain analysis of correlation between EEG-EMG and EMG-EMG was then performed. Coherence estimates are bounded measures of association defined over the range of $[0,1]$; cumulant density estimates are not bounded, and phase is defined over the range $[-\pi,+\pi]$. For the present data, coherence estimates quantify the strength and range of frequencies of the common rhythmic activity between the motor cortex and the working muscles. The cumulant density function provides an unbounded time- 
domain representation of the correlation structure analogous to the cross-correlogram Halliday et al. 1995.

The final analysis was made with estimates of pooled coherence in the frequency domain and estimates of pooled cumulant density in the time domain with an inclusion of an upper confidence limit for coherence estimates and an upper and lower confidence limit for cumulant density estimates Amjad et al. 1997, Halliday et al. 1995. Application of coherence to EMG during locomotion and the assumptions underlying this type of analysis are discussed in Halliday et al. 2003. In addition, a directionality analysis was used to decompose coherence estimates into forward and reverse components. This was done through application of a novel non-parametric approach Halliday 2015, Halliday et al. 2016 where ordinary coherence estimates are decomposed into forward, reverse and zero-lag components. The decomposition is applied to ordinary coherence, estimated from discrete Fourier transforms, and does not require any additional models (for example autoregressive) of the signals to be validated and used. The decomposition is achieved by using an optimal Minimum Mean Square Error (MMSE) pre-whitening of the two signals prior to coherence analysis. After this the coherence is given by the magnitude squared of the MMSE prewhitened cross spectrum without the need to normalize using the individual auto spectra, which are now identically 1 after MMSE pre-whitening. An inverse Fourier transform applied to the MMSE pre-whitened cross spectrum generates a time domain correlation function which is free from any contributions from the autocorrelation of either signal. From this time domain correlation function directionality is derived by considering all values at negative lag, the zero-lag value, and all values at positive lags which quantify the interaction in the reverse, zero-lag, and forward directions, respectively. A separate Fourier transform for each of these three components generates factors which allow the original coherence to be decomposed into reverse, zero-lag and forward components. In this study, we represent the forward and reverse components as a percentage of the total overall correlation. This total overall correlation, or $\mathrm{R}^{2}$ value, gives an indication of the overall strength of correlation and is calculated as the mean coherence across a specific frequency range. We chose a relatively broad frequency range $(5-50 \mathrm{~Hz})$ here to ensure that relevant frequency ranges with significant coherence were included in all cases. Since the $\mathrm{R}^{2}$ value is a cumulative measure it is minimally influenced when coherence is low and will mainly reflect frequency ranges with significant coherence. The directionality analysis is used to quantify the balance between efferent and afferent contributions to corticospinal coherence during locomotion - we equate 
efferent contributions with the forward component of coherence, and afferent contributions with the reverse component of coherence.

Two types of estimates were considered in this study and were constructed using different data segment durations. The first analysis characterizes the interactions between EEG and rectified EMG and between the two rectified EMG signals during the stance phase, where activity in the plantar flexor muscles is most pronounced. This used segments of $400 \mathrm{~ms}$ duration, which started $200 \mathrm{~ms}$ after each heel contact. The second analysis used a sliding time window with fixed duration $(250 \mathrm{~ms})$ and a variable offset from each heel contact. The offset was varied from 0 to $600 \mathrm{~ms}$ in increments of $10 \mathrm{~ms}$. This provided a time-frequency decomposition of the signals during locomotion and was used to characterize changes in correlation over the gait cycle. Undertaking a time-frequency analysis in this manner using a spectrogram based approach involving short segment Fourier transforms allowed the directionality analysis to be applied to the same segments of data. Using $400 \mathrm{~ms}$ duration data segments gives a frequency resolution of $2.5 \mathrm{~Hz}$, and a $250 \mathrm{~ms}$ data window gives a frequency resolution of $4 \mathrm{~Hz}$ in spectral estimates. The setting of confidence limits on parameter estimates to assess statistical significance is described in the cited references. Results from the first analysis, $400 \mathrm{~ms}$ data window, are shown below as line plots (Fig. 2). Results from the second time-frequency analysis, using $250 \mathrm{~ms}$ data windows, are illustrated as heat maps in the time-frequency plane, where time refers to the offset from the heel trigger to the start of the $250 \mathrm{~ms}$ data window. For time-frequency coherence estimates the $\mathrm{y}$-axis is frequency, in Hz. For the cumulant density estimates both axes are labelled as time, the x-axis is the time offset, as in the coherence estimates, the y-axis is the time lag, in ms. This time lag can be interpreted in the same manner as the time lag in a conventional cumulant or crosscovariance density estimate.

In the control study, headplots were constructed in Matlab using the EEGlab software toolbox (EEGlab 14.1.1b). The data was referenced to a frontal electrode and band pass filtered (5$1000 \mathrm{~Hz}$ ) and the plots were constructed for the sum of the coherence in the $30-65 \mathrm{~Hz}$ frequency band using a window of $500 \mathrm{~ms}$ starting at $200 \mathrm{~ms}$ after heel-strike (Fig. 2).

\section{Results}

Fig. 2 illustrates pooled coherence in the $30-64 \mathrm{~Hz}$ frequency band from 10 subjects in the control experiment. For both MG and SOL there is a clear peak of corticomuscular coherence directly over the $\mathrm{Cz}$ electrode. Coherence was larger for $\mathrm{Cz}$ than for any of the other 
electrode positions. This confirms that $\mathrm{Cz}$ was the optimal electrode placement for measuring corticomuscular coherence between EEG and EMG from the plantar flexors muscles (MG and SOL).

Fig. 3 illustrates pooled data from all 11 subjects. The analysis was performed for the $400 \mathrm{~ms}$ data window where EMG activity was observed in the SOL and MG muscles in the stance phase during gait. Coherence was calculated for pairwise recordings from Cz-SOL (Fig. 3AD), Cz-MG (Fig. 3 E-H) and SOL-MG (Fig. 3 I-L). For all combinations, significant coherence was observed at low frequencies. This very low frequency coherence likely reflects modulation of the envelope of the EMG during the gait cycle Halliday et al. 2003. Hansen et al. 2005. For the paired SOL-MG recordings, significant coherence was observed over a large range of frequencies up to around $50 \mathrm{~Hz}$ with two distinct bands of coherence at 10-20 Hz and 25-45 Hz (Fig. $3 \mathrm{~K}$ ). Similar coherence bands were not observed for the paired cortical and muscular recordings, but significant coherence was observed around $10 \mathrm{~Hz}$ and 40-45 Hz for the paired Cz-SOL recordings (Fig. $3 \mathrm{C}$ ). Coherence was more variable for the Cz-MG recording, but small significant peaks could be discerned at several frequencies between 10 and $50 \mathrm{~Hz}$ (Fig. $3 \mathrm{G}$ ).

The cumulant density estimates are shown in Fig. 3D (Cz-SOL), Fig. 3H (Cz-MG) and Fig. 3L (SOL-MG). The estimates for Cz-SOL and Cz-MG are similar and dominated by a broad peak outside the confidence band, which lasts for the full duration of the available time lags. This feature is likely to be related to the strong low frequency component seen in the coherence, reflecting the modulation of EMG, as SOL and MG are active during stance. It is difficult to discern any other systematic features in these two cumulant density estimates, possibly because they are masked by the dominant low frequency modulation of EMG. The cumulant density for SOL-MG (Fig. 3L) has a similar broad peak, but in this case a narrow central peak around zero lag is discernable. This is likely to reflect the common rhythmic modulation of motor units between the muscles as highlighted by the $10-20 \mathrm{~Hz}$ and $25-45 \mathrm{~Hz}$ coherence peaks in Fig. 3K. A similar central peak has been observed in paired TA-TA recordings during treadmill locomotion Halliday et al. 2003.

One way to reduce the dominant low frequency modulation of the EMG during gait is to use a shorter duration analysis window. The trade-off is increased frequency resolution and the need to repeat the analysis using a range of offsets to cover the period with EMG activity. Such an analysis using a $250 \mathrm{~ms}$ data window with a range of offsets is illustrated in Fig. 4, where the magnitude of coherence at different frequencies has been color coded and plotted 
against the offset following ground contact in the gait cycle (Fig. 4A-C). Around ground contact some coherence occurred for both the paired EEG and EMG recordings and the paired EMG recordings. Since there was only little EMG activity at this time, this coherence is likely due to artefacts related to the impact of the foot with the ground. Our analysis was therefore restricted to the period from $200 \mathrm{~ms}$ after ground contact. Simultaneously with the onset of EMG activity a distinct band of coherence between $\mathrm{Cz}$ and SOL was seen at frequencies around $40 \mathrm{~Hz}$ (i.e. in the gamma band; Fig. 4A). A similar distinct band of coherence was also observed for the paired MG and SOL recordings, however additional coherence could also be observed at lower frequencies (Fig. 3C). The Cz-SOL coherence in the gamma band disappeared when the SOL EMG activity began to decrease at the end of the stance phase. The MG-SOL coherence decreased somewhat earlier. The paired Cz-MG recording also showed coherence in the gamma band during the stance phase but it was less distinct than for Cz-SOL (Fig. 4B). In addition, coherence at lower and higher frequencies was observed especially around the time of push-off.

For the most part of the stance phase no distinct feature was observed in the cumulant density function for either the paired Cz-MG or Cz-SOL recordings (Fig. 5A-B). However, around the time of push-off and the subsequent toe lift (i.e. around $500 \mathrm{~ms}$ after ground contact) a relatively broad trough (marked in blue) with a center at a lag of around $-25 \mathrm{~ms}$ was seen. On either side of this trough distinct peaks at lags of around 75-80 ms were seen (Fig. 5A-B). This is consistent with a periodic component of around $6 \mathrm{~Hz}$, which can be seen in the coherence in Fig. 3C, where the maximum coherence is at this frequency and peaks around $500 \mathrm{~ms}$ offset.

\section{Directionality estimates}

Fig. 5C-D illustrates the results from the directionality analysis calculated using the same data segmentation regime as in Fig. 5A-B. Each panel shows the change in three quantities with increasing offset from heel trigger. The top panel (black) shows the overall scalar measure of correlation between the EEG and each EMG. This indicates how the overall strength of correlation changes in time. This measure is referred to as $\mathrm{R}^{2}$ in Halliday 2015. The lower panels show the percentage of the coherence in the forward (red) and reverse (blue) directions, respectively. We interpret the forward direction as reflecting descending, or efferent contributions and the reverse direction as reflecting ascending or afferent contributions to the coherence. Around the same time as the significant features in the cumulant estimates in Fig. 5A-B reach a maximum, the $\mathrm{R}^{2}$ value calculated over the 
frequency range 5-50 Hz reached a peak (upper black graphs in Fig. $5 \mathrm{C}-\mathrm{D}$ ), indicating maximal overall coherence at this time. Analysis of the directionality of coherence revealed a forward directionality (i.e. cortex leads the muscle) in the middle of the stance phase from the initiation of EMG activity (compare red (forward directionality) and blue lines (reverse directionality) in lower graphs in Fig. 5 C-D). Just prior to (SOL) or at the same time (MG) as the maximal $\mathrm{R}^{2}$ value no clear dominant directionality was observed, with roughly equal contributions in the forward and reverse directions. However, around $500 \mathrm{~ms}$ after heel contact (i.e. around the time of push off - Fig. 3), the SOL muscle was found to lead the cortex (backward directionality; blue line in Fig. 5D), whereas cortex continued to lead the MG muscle (forward directionality; red line in Fig. 5C).

\section{Discussion}

We have shown in this study that EEG recorded from the leg area of the motor cortex and EMG recorded from ankle plantar flexor muscles show coupled gamma oscillations in the stance phase during treadmill walking. Since the EEG appeared to lead the EMG activity based on directionality analysis, we suggest that these findings support that activity of corticospinal tract cells contribute to the activation of plantar flexor muscles in the stance phase of gait. Corticospinal activity may thereby play an important role in forward propulsion during gait.

This is to our knowledge the first study to demonstrate significant coherence between motor cortex and plantar flexor muscle activity during gait and analyze the directional components of this interaction. Corticomuscular coherence has previously been described for the Tibialis anterior muscle in the swing phase during gait (Petersen et al. 2012). A direct comparison with that study is not possible, as different EEG equipment and different subjects were involved. It is notable that the corticomuscular coherence observed in the present study for the plantar flexors had a higher frequency (close to $40 \mathrm{~Hz}$ as compared to close to $30 \mathrm{~Hz}$ for the Tibialis anterior muscle in Petersen et al. (2012)) and a much lower magnitude in the present study as compared to the study by Petersen et al. (2012). The latter observation is consistent with a relatively low contribution of corticospinal input to ankle plantar flexors as compared to ankle dorsiflexors, as previously suggested from both animal and human experiments Capaday et al. 1999, Drew et al. 1996. However, it may also more simply reflect differences in the firing behavior of the neurons involved in generating corticomuscular coherence for the two muscle groups. Corticomuscular coherence requires relatively stable discharges within restricted frequency bands in both descending and 
ascending pathways Witham et al. 2011. Corticospinal neurons projecting to ankle plantar flexors may show a more dynamic modulation of firing rates during stance than the equivalent for ankle dorsiflexors and thereby diminish the likelihood of detecting corticomuscular coherence. In addition, the contact with the ground may elicit sensory feedback that may interfere with the rhythmic oscillations in the network (i.e. sensory desynchronization).

It is also noteworthy that the coherence observed between the MG and SOL EMG activities was seen at a somewhat lower frequency $(30-40 \mathrm{~Hz})$ than the corticomuscular coherence. This is similar to what has been observed for the Tibialis anterior muscle where coherence between different motor unit populations during gait tends to be observed below $30 \mathrm{~Hz}$ and even as low as $15-20 \mathrm{~Hz}$ in the swing phase during gait. The involvement of different frequency bands is indicative of possible different mechanisms or non-linear interactions. It would require alternative non-linear analysis techniques, which have so far not been validated sufficiently for EMG signals, to address this further Cekic et al. 2018.

Coherence at low frequencies (alpha band; $5-15 \mathrm{~Hz}$ ) between pairs of motor unit populations has been reported previously for other muscles and other tasks, although corticomuscular coherence is generally not seen at such frequencies Halliday et al. 2003, Petersen et al. 2012, Semmler et al. 2004). This was also the case in the present study. One possibility is that this alpha band coherence is generated from the motor cortex similar to the coherence at higher frequencies as some studies have been able to detect significant corticomuscular coherence in the alpha range Raethjen et al. 2002. There is also evidence of oscillatory activity in cerebellar-thalamus-motor cortex circuitries recorded by electrodes inserted for deep brain stimulation during surgery Marsden et al. 2000. This oscillatory activity, which is likely related also to physiological tremor, may be transmitted via the motor cortex or other descending tracts to the spinal motor neurons and cause the observed coherence between the motor unit populations McAuley et al. 1997. In all likelihood this requires oscillatory activity in the alpha frequency band of last order neurons with branches to both populations of motor units similar to what has been shown for coherence at higher frequencies Conway et al. 1995. The failure to detect corticomuscular coherence in the alpha band in this and other studies may also suggest that the circuitries, which are responsible for generating the intermuscular coherence in the alpha band involve a more indirect pathway from the motor cortex than the pathways responsible for coherence at higher frequencies. Other sources that influence corticospinal coherence include spinal interneurons. Matsuya and colleagues reported a close connection between recurrent inhibition and peak soleus corticomuscular 
coherence during a maintained plantarflexion. They thus reported a positive correlation between the amount of corticospinal coherence between $\mathrm{Cz}$ EEG and SOL and the level of recurrent inhibition indicating that spinal mechanisms indeed modulate the corticomuscular coherence. In the present study, the source of the oscillatory activity cannot be isolated and both cortical and spinal mechanisms most likely contribute and modulate the observed coherence Matsuya et al. 2017.

\section{Comparison to static contraction}

The observed corticomuscular coherence during gait is several magnitudes lower than that, which has been reported during static plantar flexion Perez et al. 2007, Ushiyama et al. 2010. Although Petersen et al (2012) found reproducible corticomuscular coherence for the Tibialis anterior muscle in the swing phase of gait, the magnitude of this coherence was also several magnitudes lower than what is normally reported for static dorsiflexion Petersen et al. 2012. This is not surprising since coherence between cortex and muscle will require relatively constant firing rates of the corticospinal neurons within a narrow band of frequencies, which is unlikely to occur to any large extent during a dynamic task like walking. Recordings from the cat motor cortex during gait or from monkey corticospinal neurons during hand movements rather suggest that the corticospinal neurons discharge at greatly varying rates and are only active for relatively brief periods of time. The fact that we have been able to detect significant corticomuscular coherence despite of the dynamic nature of the task is therefore noteworthy. There are a number of factors that could explain the low levels of coherence in this study. The estimates are pooled across subjects so may well be affected by variability between subjects. The task is highly dynamic in nature, this is clear from the time frequency and directionality plots which indicate changes in the strength of correlation and in the directional components can occur over time scales of around $200 \mathrm{~ms}$. The analysis is based on coherence estimation which assumes linear interactions, application of nonlinear analysis may provide further insight into the complex interactions between cortical and spinal neurons Cunningham et al. 2004.

\section{Directionality}

Application of the non-parametric directionality analysis provides additional insight into the cortical control of muscles during locomotion. The main finding is that the directionality is predominantly in the forward (descending) direction during the early part of stance, with equal contributions from descending and ascending pathways during mid stance and 
switching again to a preferred direction in later stance. This preferred direction prior to toeoff is forward (descending) for MG and reverse (ascending) for SOL. The reason for the difference directionality prior to toe-off is unclear, but may reflect the different roles of sensory inputs in determining the transition from stance to swing.

In hand muscles both forward and reverse components of the corticomuscular coherence have been observed Witham et al. 2011. The authors speculate that the observed coherence might represent a loop integrating the sensory feedback with the motor command to optimize the intended movement or promote a more stable motor state. In both cases a prominent role for sensory feedback in generating the oscillatory activity is suggested Witham et al., 2011. There are probably differences in the control strategy of the hand muscles during a ramp and hold task as used in the study of Witham and coworkers and the much more dynamic muscles activation required to walk on a motorized treadmill. In the present study both forward and reverse components of coherence were present surporting a role for both sensory feedback and direct corticospinal activity in contributing to the observed coherence during the stance phase and push-off of normal walking.

\section{Functional and clinical significance}

Ankle plantar flexor muscles are of fundamental importance for human gait since they exert their action directly on the supporting ground and are in a position to both support the body against gravity and help to propel the body forward by providing a forward thrust at push-off Winter 1983. Despite a long-standing debate there is no general consensus as to whether their main role is in support or forward propulsion Honeine et al. 2013, Neptune et al. 2001. According to the controlled roll-off theory the plantarflexors mainly contribute to ensure stability, while the body rotates over the stance leg Honeine et al. 2013, whereas the pushoff theory suggests that the plantarflexors actively push the body forward late in stance Neptune and Kautz 2001, Winter 1983. It has also been suggested that the primary role of the plantar flexors would be to propel the limb into flexion and that the main forward propulsion is generated later as energy from the swing leg is transferred to the trunk Hof et al. 1992, Meinders et al. 1998. Unfortunately, there are still several limitations to current forward kinematics modeling and inverse dynamics calculations obtained from biomechanical gait analysis that limit our ability to address the details of this question at the moment.

Using a complementary approach, namely assisting push-off using a robotic ankle-foot orthosis, Sawicki and Ferris showed a reduction in SOL EMG when the assistance was 
activated, confirming a role of plantarflexors during this phase of the gait cycle Sawicki and Ferris 2008. In line with this, Sinkjær and colleagues showed a decrease in SOL activity when unloading the plantar flexors during the stance phase of normal walking. These findings show that sensory feedback contributes to plantar flexor activity during gait and plays a functional role during gait. Sinkjaer et al. 2000.

Several studies have also demonstrated a relation between weakness of plantar flexors, reduced push-off velocity and forward propulsion in patients with central motor lesions Mueller et al. 1995, Nadeau et al. 1999, Olney et al. 1990. This is well in line with our observation of significant coupling between motor cortex and plantar flexor muscle activity.

\section{Conclusion}

We have demonstrated that it is possible to detect significant corticomuscular coherence between the motor cortex and ankle plantar flexor muscles in the stance phase during treadmill walking. This suggests that activity in corticospinal neurons contribute to the activity of plantar flexor muscles during stance and we propose that the motor cortex activity contributes to forward propulsion during gait.

\section{REFERENCES}

Amjad AM, Halliday DM, Rosenberg JR, Conway BA (1997) An extended difference of coherence test for comparing and combining several independent coherence estimates: theory and application to the study of motor units and physiological tremor J Neurosci Methods 73:6979

Barthelemy D, Knudsen H, Willerslev-Olsen M, Lundell H, Nielsen JB, Biering-Sorensen F (2013) Functional implications of corticospinal tract impairment on gait after spinal cord injury Spinal cord 51:852-856 doi:10.1038/sc.2013.84

Bramble DM, Lieberman DE (2004) Endurance running and the evolution of Homo Nature 432:345352 doi:10.1038/nature03052

Brouwer B, Parvataneni K, Olney SJ (2009) A comparison of gait biomechanics and metabolic requirements of overground and treadmill walking in people with stroke Clinical biomechanics 24:729-734 doi:10.1016/j.clinbiomech.2009.07.004

Capaday C, Lavoie BA, Barbeau H, Schneider C, Bonnard M (1999) Studies on the corticospinal control of human walking. I. Responses to focal transcranial magnetic stimulation of the motor cortex Journal of neurophysiology 81:129-139

Cappellini G, Ivanenko YP, Dominici N, Poppele RE, Lacquaniti F (2010) Migration of motor pool activity in the spinal cord reflects body mechanics in human locomotion Journal of neurophysiology 104:3064-3073 doi:10.1152/jn.00318.2010

Cekic S, Grandjean D, Renaud O (2018) Time, frequency, and time-varying Granger-causality measures in neuroscience Stat Med 37:1910-1931 doi:10.1002/sim.7621

Christensen LO, Petersen N, Andersen JB, Sinkjaer T, Nielsen JB (2000) Evidence for transcortical reflex pathways in the lower limb of man Progress in neurobiology 62:251-272 
Conway BA, Halliday DM, Farmer SF, Shahani U, Maas P, Weir Al, Rosenberg JR (1995)

Synchronization between motor cortex and spinal motoneuronal pool during the performance of a maintained motor task in man The Journal of physiology 489 ( Pt 3):917924

Cunningham MO, Halliday DM, Davies CH, Traub RD, Buhl EH, Whittington MA (2004) Coexistence of gamma and high-frequency oscillations in rat medial entorhinal cortex in vitro The Journal of physiology 559:347-353 doi:10.1113/jphysiol.2004.068973

Dietz V (2010) Behavior of spinal neurons deprived of supraspinal input Nature reviews Neurology 6:167-174 doi:10.1038/nrneurol.2009.227

Drew T, Jiang W, Kably B, Lavoie S (1996) Role of the motor cortex in the control of visually triggered gait modifications Can J Physiol Pharmacol 74:426-442

Eidelberg E, Walden JG, Nguyen LH (1981) Locomotor control in macaque monkeys Brain : a journal of neurology 104:647-663

Everaert DG, Thompson AK, Chong SL, Stein RB (2010) Does functional electrical stimulation for foot drop strengthen corticospinal connections? Neurorehabilitation and neural repair 24:168177 doi:10.1177/1545968309349939

Fedirchuk B, Nielsen J, Petersen N, Hultborn H (1998) Pharmacologically evoked fictive motor patterns in the acutely spinalized marmoset monkey (Callithrix jacchus) Experimental brain research 122:351-361

Geertsen SS, Kirk H, Lorentzen J, Jorsal M, Johansson CB, Nielsen JB (2015) Impaired gait function in adults with cerebral palsy is associated with reduced rapid force generation and increased passive stiffness Clinical neurophysiology : official journal of the International Federation of Clinical Neurophysiology 126:2320-2329 doi:10.1016/j.clinph.2015.02.005

Halliday DM (2015) Nonparametric directionality measures for time series and point process data J Integr Neurosci 14:253-277 doi:10.1142/S0219635215300127

Halliday DM, Conway BA, Christensen LO, Hansen NL, Petersen NP, Nielsen JB (2003) Functional coupling of motor units is modulated during walking in human subjects Journal of neurophysiology 89:960-968 doi:10.1152/jn.00844.2002

Halliday DM, Farmer SF (2010) On the need for rectification of surface EMG Journal of neurophysiology 103:3547; author reply 3548-3549 doi:10.1152/jn.00222.2010

Halliday DM, Rosenberg JR (2000) On the application, estimation and interpretation of coherence and pooled coherence J Neurosci Methods 100:173-174

Halliday DM, Rosenberg JR, Amjad AM, Breeze P, Conway BA, Farmer SF (1995) A framework for the analysis of mixed time series/point process data--theory and application to the study of physiological tremor, single motor unit discharges and electromyograms Prog Biophys Mol Biol 64:237-278

Halliday DM, Senik MH, Stevenson CW, Mason R (2016) Non-parametric directionality analysis Extension for removal of a single common predictor and application to time series $\mathrm{J}$ Neurosci Methods 268:87-97 doi:10.1016/j.jneumeth.2016.05.008

Hamacher D, Herold F, Wiegel P, Hamacher D, Schega L (2015) Brain activity during walking: A systematic review Neuroscience and biobehavioral reviews 57:310-327 doi:10.1016/j.neubiorev.2015.08.002

Hansen NL, Conway BA, Halliday DM, Hansen S, Pyndt HS, Biering-Sorensen F, Nielsen JB (2005) Reduction of common synaptic drive to ankle dorsiflexor motoneurons during walking in patients with spinal cord lesion Journal of neurophysiology 94:934-942 doi:10.1152/jn.00082.2005

Hegarty AK, Kurz MJ, Stuberg W, Silverman AK (2016) Changes in Mobility and Muscle Function of Children With Cerebral Palsy After Gait Training: A Pilot Study Journal of applied biomechanics:1-24 doi:10.1123/jab.2015-0311

Hof AL, Nauta J, van der Knaap ER, Schallig MA, Struwe DP (1992) Calf muscle work and segment energy changes in human treadmill walking Journal of electromyography and kinesiology: 
official journal of the International Society of Electrophysiological Kinesiology 2:203-216 doi:10.1016/1050-6411(92)90024-D

Honeine JL, Schieppati M, Gagey O, Do MC (2013) The functional role of the triceps surae muscle during human locomotion PloS one 8:e52943 doi:10.1371/journal.pone.0052943

Kiehn O (2016) Decoding the organization of spinal circuits that control locomotion Nature reviews Neuroscience 17:224-238 doi:10.1038/nrn.2016.9

Knarr BA, Kesar TM, Reisman DS, Binder-Macleod SA, Higginson JS (2013) Changes in the activation and function of the ankle plantar flexor muscles due to gait retraining in chronic stroke survivors Journal of neuroengineering and rehabilitation 10:12 doi:10.1186/1743-0003-1012

la Fougere C et al. (2010) Real versus imagined locomotion: a [18F]-FDG PET-fMRI comparison Neurolmage 50:1589-1598 doi:10.1016/j.neuroimage.2009.12.060

Marsden JF, Ashby P, Limousin-Dowsey P, Rothwell JC, Brown P (2000) Coherence between cerebellar thalamus, cortex and muscle in man: cerebellar thalamus interactions Brain : a journal of neurology 123 ( Pt 7):1459-1470

Matsuya R, Ushiyama J, Ushiba J (2017) Inhibitory interneuron circuits at cortical and spinal levels are associated with individual differences in corticomuscular coherence during isometric voluntary contraction Sci Rep 7:44417 doi:10.1038/srep44417

McAuley JH, Rothwell JC, Marsden CD (1997) Frequency peaks of tremor, muscle vibration and electromyographic activity at $10 \mathrm{~Hz}, 20 \mathrm{~Hz}$ and $40 \mathrm{~Hz}$ during human finger muscle contraction may reflect rhythmicities of central neural firing Experimental brain research 114:525-541

Meinders M, Gitter A, Czerniecki JM (1998) The role of ankle plantar flexor muscle work during walking Scandinavian journal of rehabilitation medicine 30:39-46

Mueller MJ, Minor SD, Schaaf JA, Strube MJ, Sahrmann SA (1995) Relationship of plantar-flexor peak torque and dorsiflexion range of motion to kinetic variables during walking Physical therapy 75:684-693

Nadeau S, Gravel D, Arsenault AB, Bourbonnais D (1999) Plantarflexor weakness as a limiting factor of gait speed in stroke subjects and the compensating role of hip flexors Clinical biomechanics 14:125-135

Neptune RR, Kautz SA (2001) Muscle activation and deactivation dynamics: the governing properties in fast cyclical human movement performance? Exercise and sport sciences reviews 29:7680

Neptune RR, Kautz SA, Zajac FE (2001) Contributions of the individual ankle plantar flexors to support, forward progression and swing initiation during walking Journal of biomechanics 34:1387-1398

Nielsen JB (2003) How we walk: central control of muscle activity during human walking The Neuroscientist : a review journal bringing neurobiology, neurology and psychiatry 9:195-204

Nielsen JB, Sinkjaer T (2002) Afferent feedback in the control of human gait Journal of electromyography and kinesiology : official journal of the International Society of Electrophysiological Kinesiology 12:213-217

Noel M, Cantin B, Lambert S, Gosselin CM, Bouyer $\amalg$ (2008) An electrohydraulic actuated ankle foot orthosis to generate force fields and to test proprioceptive reflexes during human walking IEEE transactions on neural systems and rehabilitation engineering : a publication of the IEEE Engineering in Medicine and Biology Society 16:390-399 doi:10.1109/TNSRE.2008.926714

Olney SJ, MacPhail HE, Hedden DM, Boyce WF (1990) Work and power in hemiplegic cerebral palsy gait Physical therapy 70:431-438

Pepin A, Ladouceur M, Barbeau H (2003a) Treadmill walking in incomplete spinal-cord-injured subjects: 2 . Factors limiting the maximal speed Spinal cord 41:271-279 doi:10.1038/sj.sc.3101453 
Pepin A, Norman KE, Barbeau H (2003b) Treadmill walking in incomplete spinal-cord-injured subjects: 1. Adaptation to changes in speed Spinal cord 41:257-270 doi:10.1038/sj.sc.3101452

Perez MA, Lundbye-Jensen J, Nielsen JB (2007) Task-specific depression of the soleus H-reflex after cocontraction training of antagonistic ankle muscles Journal of neurophysiology 98:36773687 doi:10.1152/jn.00988.2007

Petersen N, Christensen LO, Nielsen J (1998) The effect of transcranial magnetic stimulation on the soleus $\mathrm{H}$ reflex during human walking The Journal of physiology 513 ( Pt 2):599-610

Petersen NT et al. (2001) Suppression of EMG activity by transcranial magnetic stimulation in human subjects during walking The Journal of physiology 537:651-656

Petersen TH, Willerslev-Olsen M, Conway BA, Nielsen JB (2012) The motor cortex drives the muscles during walking in human subjects The Journal of physiology 590:2443-2452 doi:10.1113/jphysiol.2012.227397

Preuschoft $\mathrm{H}$ (2004) Mechanisms for the acquisition of habitual bipedality: are there biomechanical reasons for the acquisition of upright bipedal posture? Journal of anatomy 204:363-384 doi:10.1111/j.0021-8782.2004.00303.x

Raethjen J et al. (2002) Corticomuscular coherence in the $6-15 \mathrm{~Hz}$ band: is the cortex involved in the generation of physiologic tremor? Experimental brain research 142:32-40 doi:10.1007/s00221-001-0914-7

Sawicki GS, Ferris DP (2008) Mechanics and energetics of level walking with powered ankle exoskeletons J Exp Biol 211:1402-1413 doi:10.1242/jeb.009241

Schubert M, Curt A, Jensen L, Dietz V (1997) Corticospinal input in human gait: modulation of magnetically evoked motor responses Experimental brain research 115:234-246

Semmler JG, Sale MV, Meyer FG, Nordstrom MA (2004) Motor-unit coherence and its relation with synchrony are influenced by training Journal of neurophysiology 92:3320-3331 doi:10.1152/jn.00316.2004

Shapiro LJ, Jungers WL (1994) Electromyography of back muscles during quadrupedal and bipedal walking in primates American journal of physical anthropology 93:491-504 doi:10.1002/ajpa.1330930408

Simonsen EB (2014) Contributions to the understanding of gait control Danish medical journal 61:B4823

Sinkjaer T, Andersen JB, Ladouceur M, Christensen LO, Nielsen JB (2000) Major role for sensory feedback in soleus EMG activity in the stance phase of walking in man The Journal of physiology 523 Pt 3:817-827

Steudel-Numbers KL (2001) Role of locomotor economy in the origin of bipedal posture and gait American journal of physical anthropology 116:171-173 doi:10.1002/ajpa.1110

Trank TV, Chen C, Smith JL (1996) Forms of forward quadrupedal locomotion. I. A comparison of posture, hindlimb kinematics, and motor patterns for normal and crouched walking Journal of neurophysiology 76:2316-2326

Ushiyama J, Takahashi Y, Ushiba J (2010) Muscle dependency of corticomuscular coherence in upper and lower limb muscles and training-related alterations in ballet dancers and weightlifters J Appl Physiol (1985) 109:1086-1095 doi:10.1152/japplphysiol.00869.2009

Wang WJ, Crompton RH (2004) The role of load-carrying in the evolution of modern body proportions Journal of anatomy 204:417-430 doi:10.1111/j.0021-8782.2004.00295.x

Watson J, Payne R, Chamberlain A, Jones R, Sellers WI (2009) The kinematics of load carrying in humans and great apes: implications for the evolution of human bipedalism Folia primatologica; international journal of primatology 80:309-328 doi:10.1159/000258646

Winter DA (1983) Energy generation and absorption at the ankle and knee during fast, natural, and slow cadences Clinical orthopaedics and related research:147-154 
Witham CL, Riddle CN, Baker MR, Baker SN (2011) Contributions of descending and ascending pathways to corticomuscular coherence in humans The Journal of physiology 589:3789-3800 doi:10.1113/jphysiol.2011.211045

Zehr EP, Stein RB (1999) What functions do reflexes serve during human locomotion? Progress in neurobiology 58:185-205 

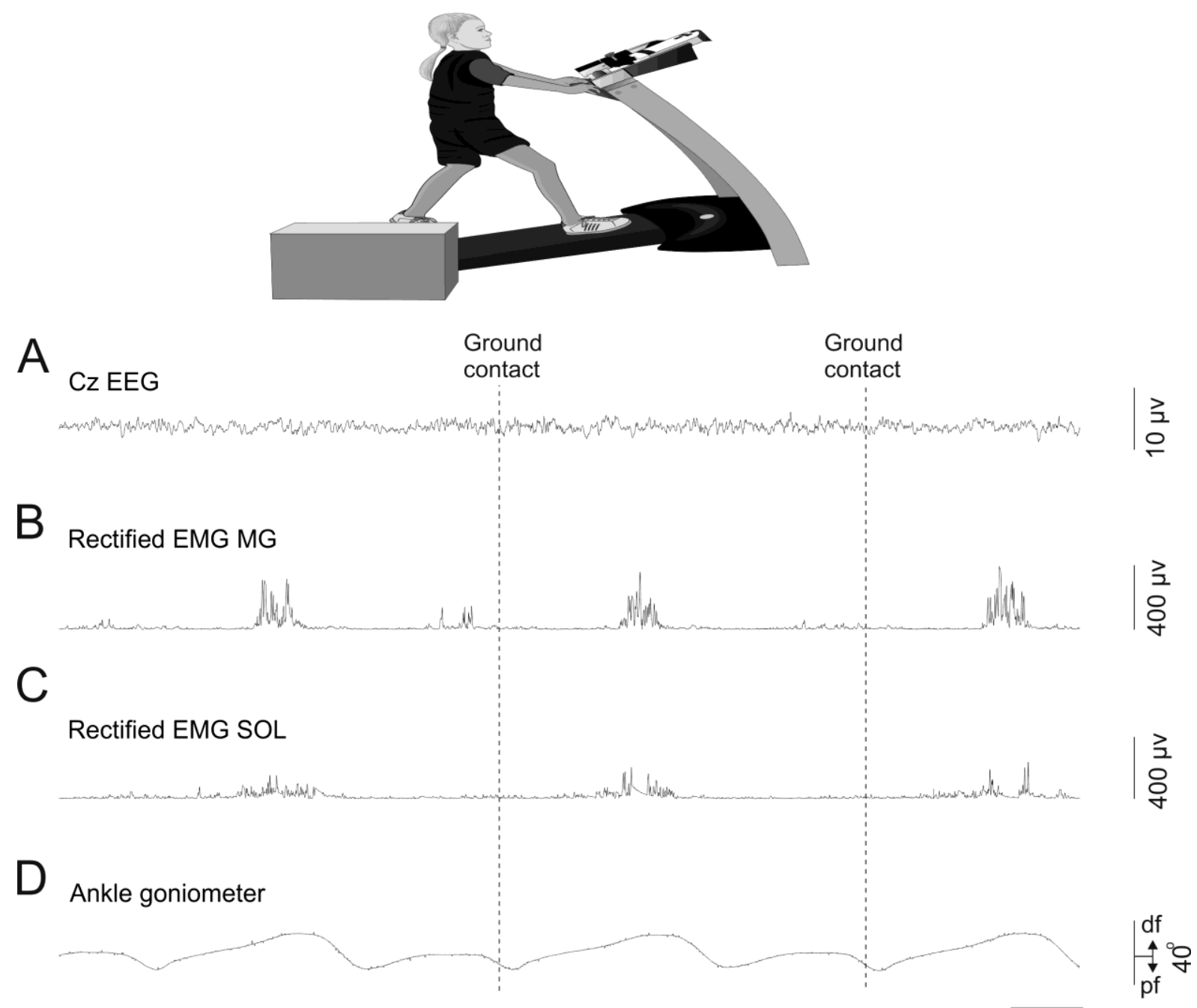

$200 \mathrm{~ms}$ 

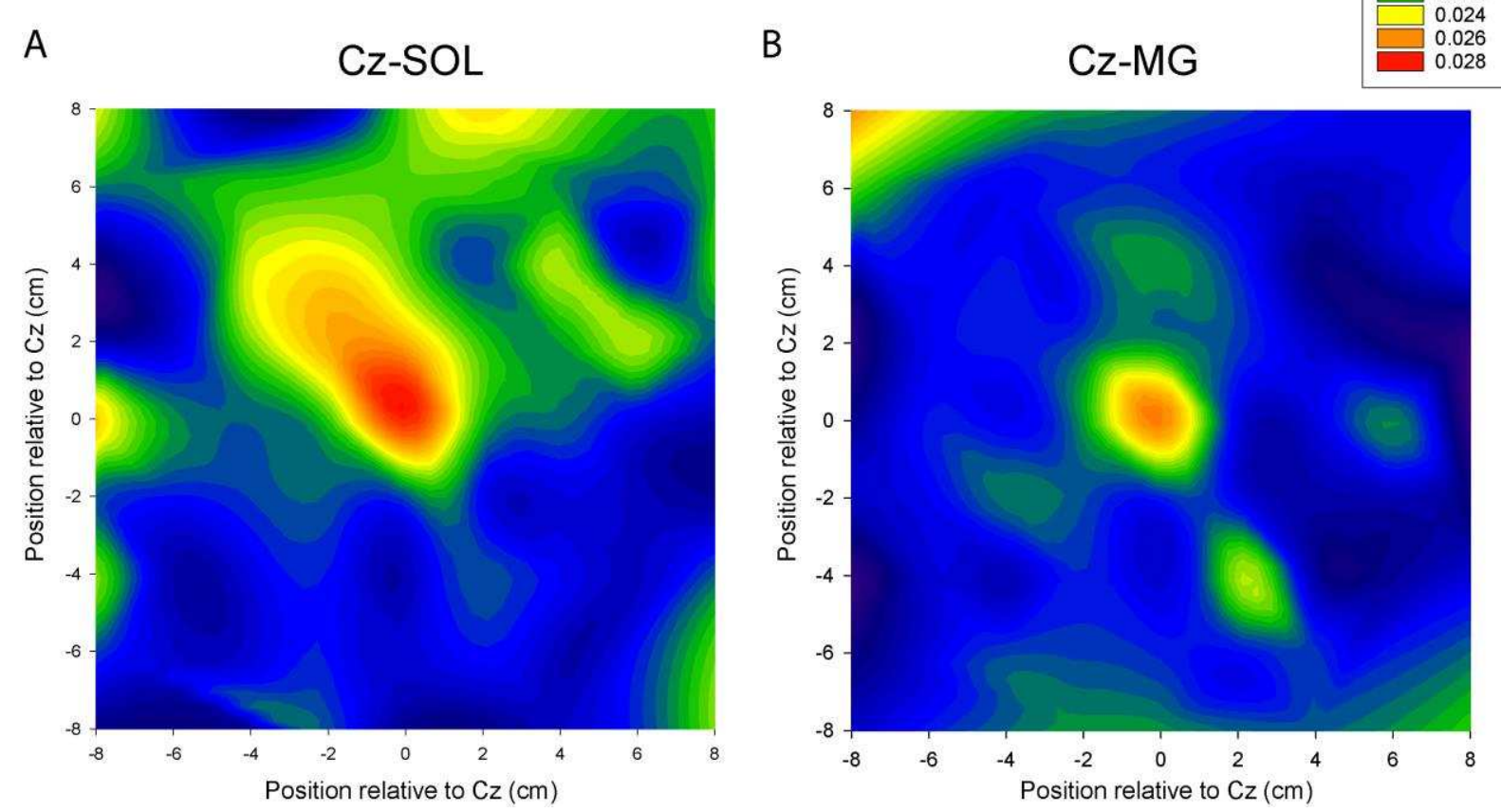


\section{$\mathrm{Cz}-\mathrm{SOL}$}

A

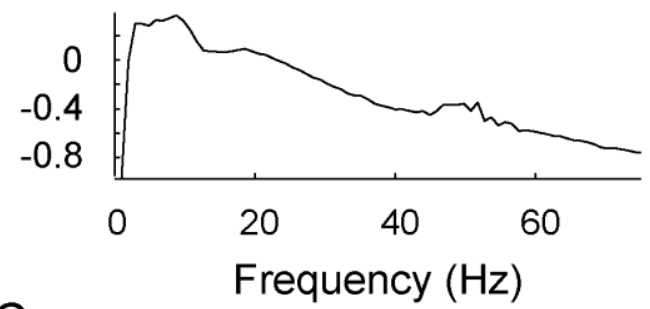

C

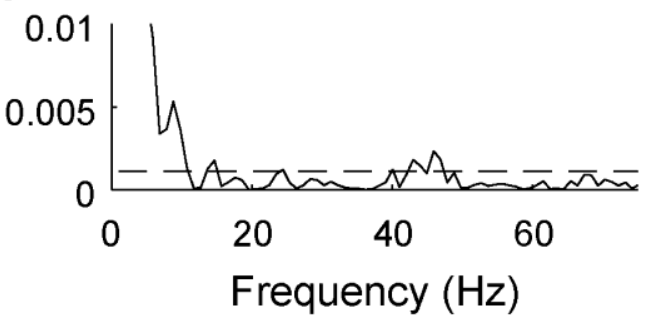

B

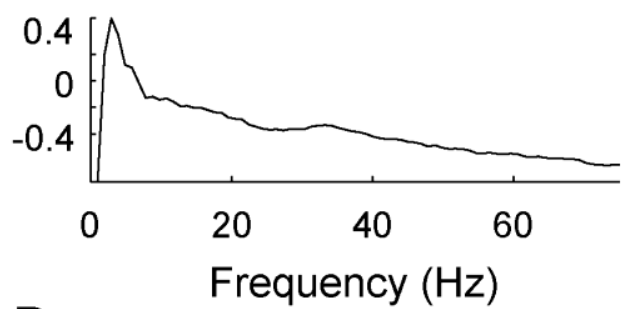

D

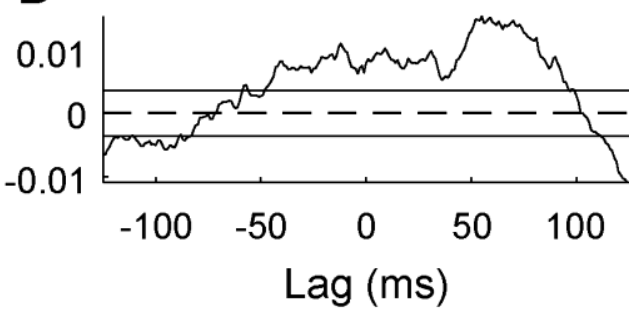

Cz-MG

E

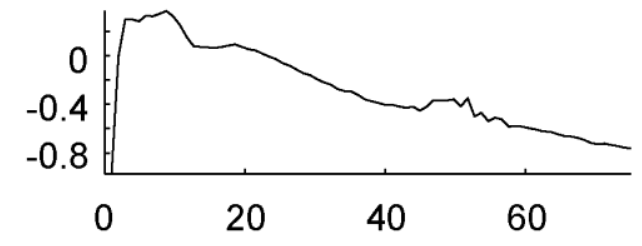

G

Frequency $(\mathrm{Hz})$

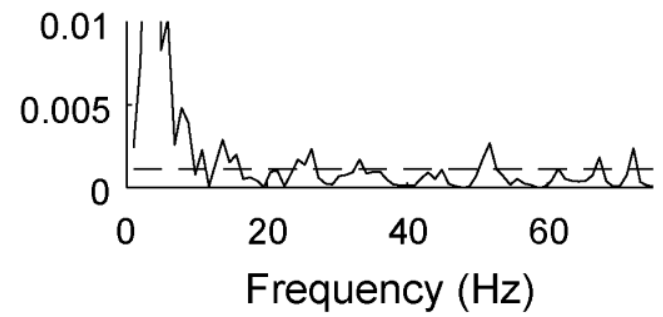

$F$

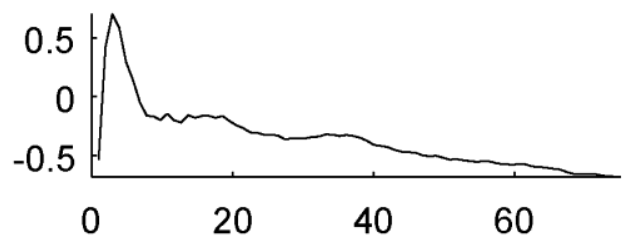

$\mathrm{H}$

Frequency $(\mathrm{Hz})$

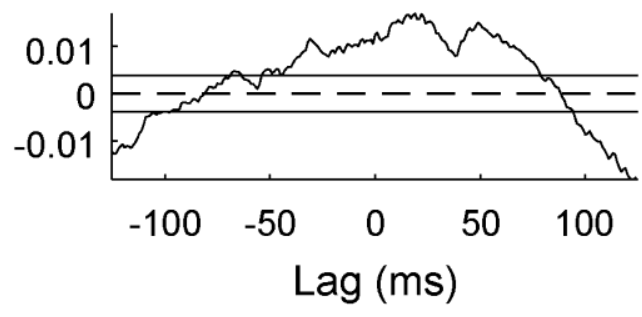

SOL-MG

I

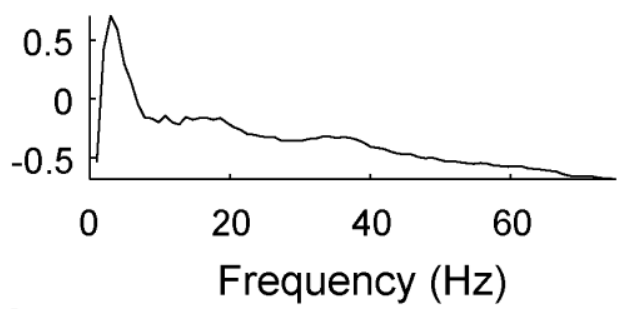

K

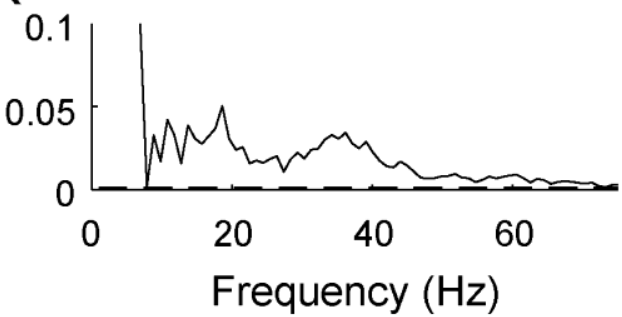

L

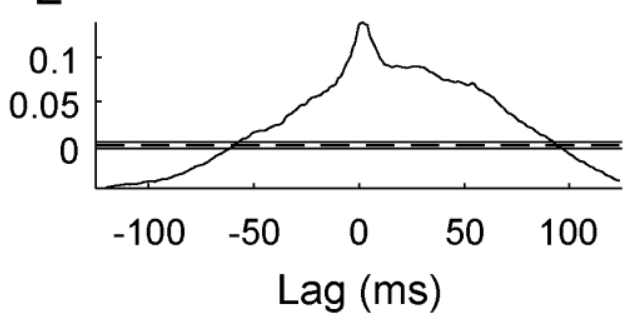



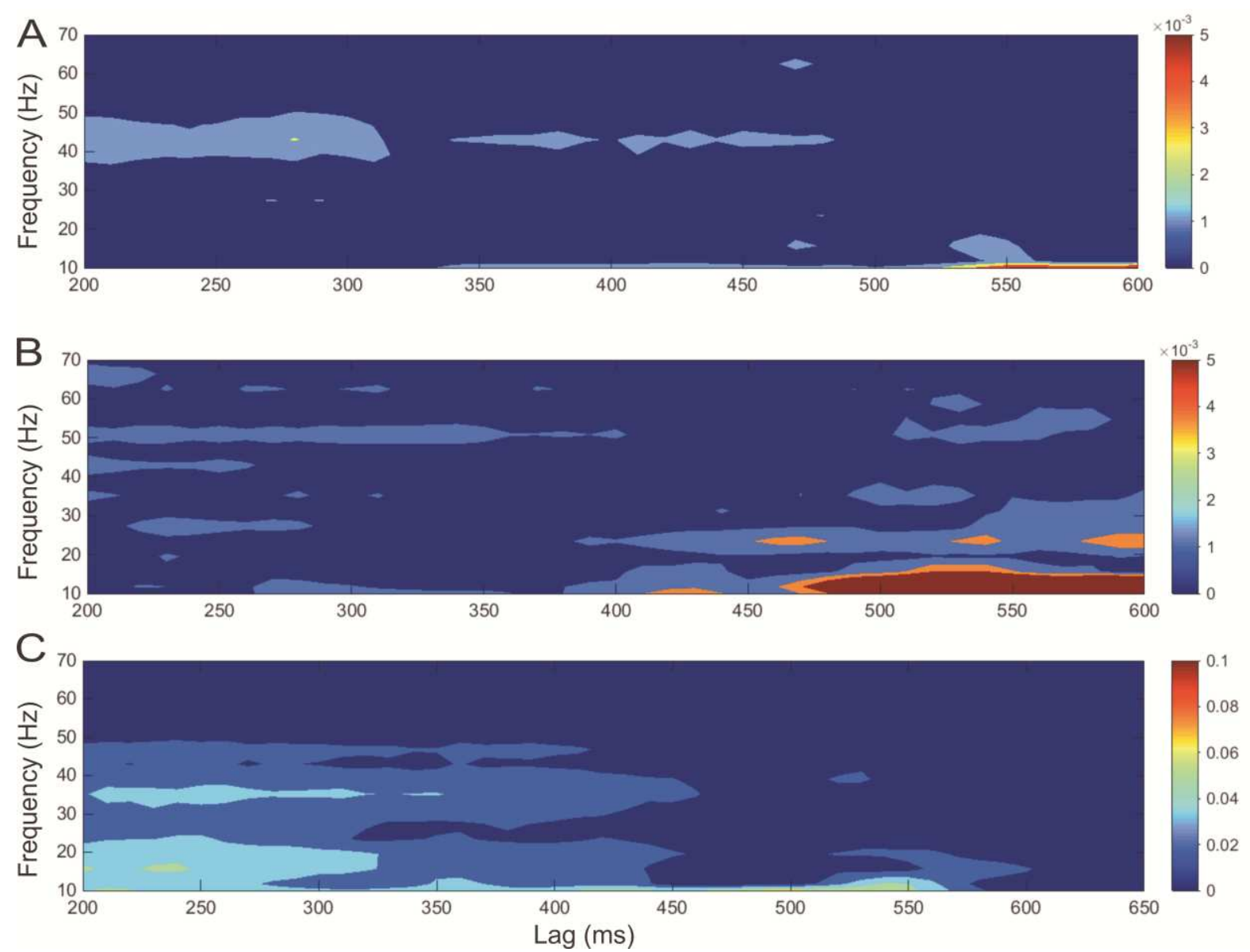


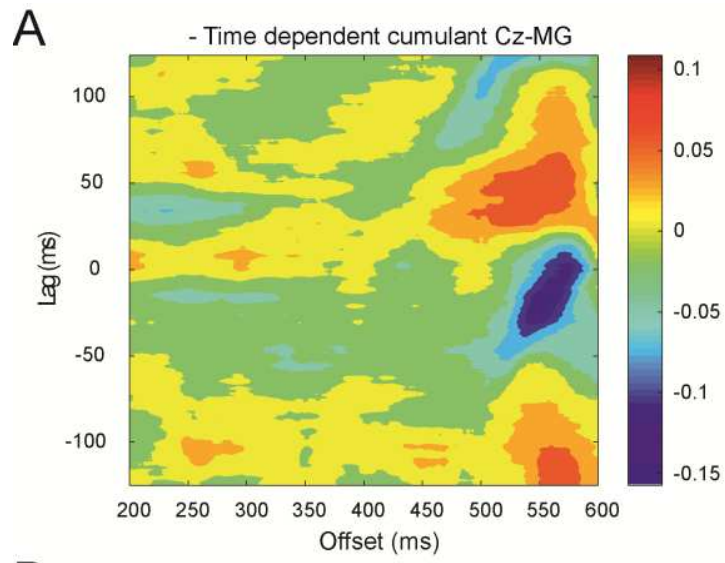

B

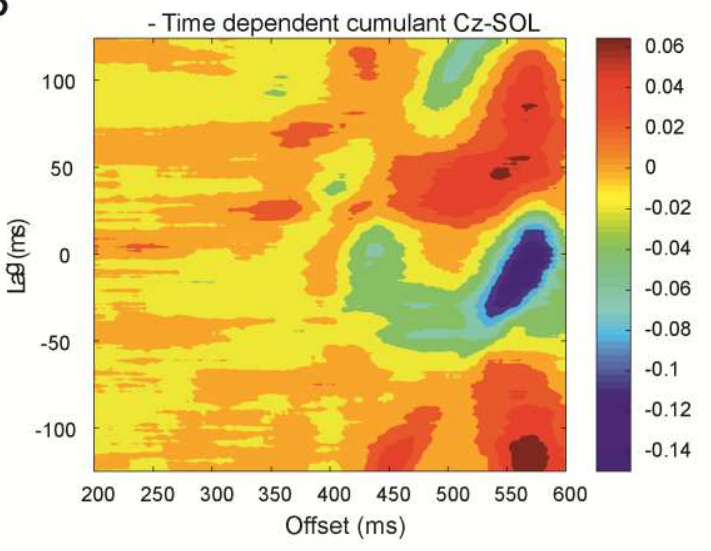

C

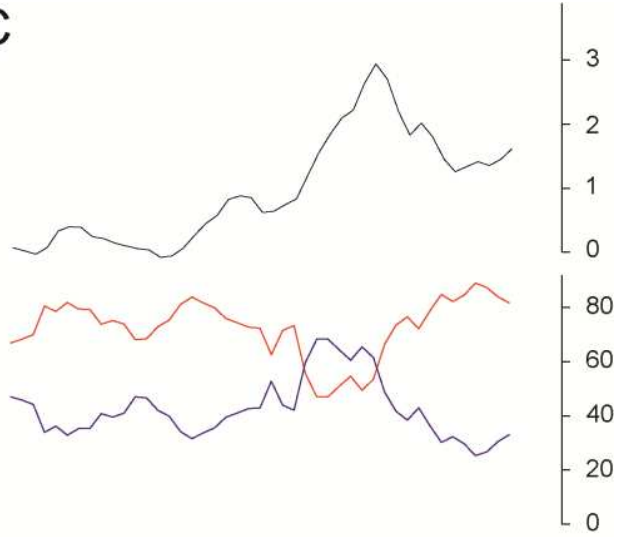

D

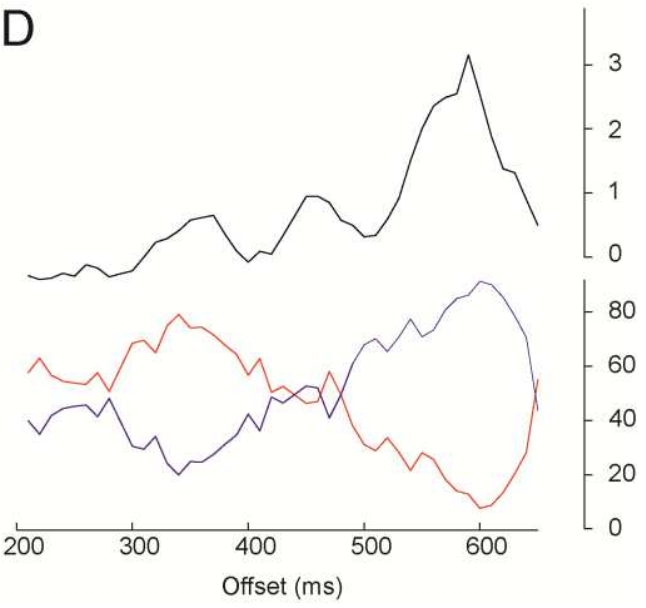

